\title{
Fractal heterogeneities in sonic logs and low-frequency scattering attenuation ${ }^{a}$
}

${ }^{a}$ Published in Geophysics, 74, no. 2, WA77-WA92, (2009)

Thomas J. Browaeys and Sergey Fomel

\begin{abstract}
Cycles in sedimentary strata exist at different scales and can be described by fractal statistics. We use von Kármán's autocorrelation function to model heterogeneities in sonic logs from a clastic reservoir and propose a nonlinear parameter estimation. Our method is validated using synthetic signals, and when applied to real sonic logs, it extracts both the fractal properties of high spatial frequencies and one dominant cycle between 2.5 and $7 \mathrm{~m}$. Results demonstrate non-Gaussian and antipersistent statistics of sedimentary layers. We derive an analytical formula for the scattering attenuation of scalar waves by 3D isotropic fractal heterogeneities using the mean field theory. Penetration of waves exhibits a high-frequency cutoff sensitive to heterogeneity size. Therefore shear waves can be more attenuated than compressional waves because of their shorter wavelength.
\end{abstract}

\section{INTRODUCTION}

Propagation of waves in heterogeneous media involves attenuation and dispersion by scattering. Theoreticians are still challenged by the phenomenon of wave propagation in random media. The mean field theory (Chernov, 1960; Karal and Keller, 1964; Uscinski, 1977) is commonly used and provides both dispersion and attenuation, depending on scattering cross-sections of the heterogeneities (Waterman and Truell, 1961; Wu and Aki, 1985; Kanaun and Levin, 2008), which are described by their statistical spatial autocorrelation. Higher order correlations have been more recently incorporated in a frequency-dependent effective medium theory (Chesnokov et al., 1998). One major advance, pointed out by Wu (1982); Wu and Aki (1985), is the restriction of the validity of the mean field formalism to low frequency. The theory in fact includes destructive interferences, caused by averaging different realizations of the random medium, and overestimates attenuation at high frequencies. Alternative solutions have been proposed to remove this artificial decoherence of the phase. Two important examples are radiative transfer theory (Wu, 1993; Haney et al., 2005) and the Rytov approximation (Rytov et al., 1989), which is more adequate than the Born approximation when phase fluctuations are important. 
In the area of seismic imaging, the layered structure of sediments led O'Doherty and Anstey (1971) to introduce the fundamental concept of stratigraphic filtering. The empirical formula postulated by O'Doherty and Anstey was demonstrated in 1D using mean field formalism (Banik et al., 1985; Resnick, 1990) and, alternately, using wave localization theory (Sheng et al., 1986; Shapiro and Zien, 1993; Shapiro and Hubral, 1999), with a recent extension to a larger frequency band for acoustic waves in 3D (Müller and Shapiro, 2001). The wave localization method utilizes phase, and logarithm of the amplitude, which have the property of self-averaging over some distance called the localization length, in a stationary random medium. These quantities are exactly the ones used in the Rytov method and avoid the phenomenon of artificial phase decoherence at high frequencies. Multiple scattering of seismic waves remains a complex and active research area.

The fractal property of subsurface heterogeneities was initially discussed by Hewett (1986) for hydrocarbon reservoirs and has been confirmed in both vertical and horizontal wells (Stefani and Gopa, 2001). The study of seismic scattering by 2D numerical wave propagation (Frankel and Clayton, 1986) demonstrates the necessity of self-similar heterogeneities for modeling both observations of coda waves and of traveltime anomalies. A $1 / f$ spectrum of heterogeneities was modeled using the von Kármán spatial autocorrelation function (von Kármán, 1948) in order to obtain a constant quality factor at high frequencies. For scatterers larger than the wavelength, multipathing was observed, whereas 3D effects were revealed to be important for scattering loss at low frequency. Gist (1994) tried to explain seismic-wave attenuation in VSP surveys by 3D scattering from fractal heterogeneities. One of the first attempts to relate the statistics of well log data to seismic scattering used wave localization theory (White et al., 1990). A more detailed study of acoustic-wave localization effects in 1D fractal media (van der Baan, 2001) shows that a constant quality factor is possible only for the $1 / f$ fractal spectrum and that localization can not occcur if the medium contains periodic layers involving resonance and violating the ergodicity assumption. Convergence of the localization effect in realistic 3D seismic surveys seems questionable. Presence of strong cycles in well log data is causing difficulties when the fractal exponent is being estimated (Dolan et al., 1998) and is commonly attributed to Milankovitch cycles (Anstey and O'Doherty, 2002a).

Further investigation of the relationship between cycles, fractal properties, and correlation lengths is necessary and low-frequency scattering theories in 3D fractal media can be appropriate for conventional seismic surveys. In this paper, we propose a nonlinear estimation method for fractal statistics of sonic-log heterogeneities using von Kármán's model. We attempt to identify different scales of the sedimentation process as proposed by O'Doherty and Anstey (1971) and Anstey and O'Doherty (2002a). The inversion captures small-scale heterogeneities while larger local cycles exist. We use the mean field theory to calculate analytical solution of low-frequency attenuation by scattering from 3D fractal heterogeneities and predict a shift of the dominant frequency with depth in seismic surveys.

The paper is organized into two parts. In the first part we present a description 
of cycles in sediments in connection with fractal statistics. The von Kármán spatial autocorrelation function is introduced, and we briefly review some features of fractal statistics. We present our estimation method, validate it on synthetic signals, apply it to our sonic-log data, and show that one can detect the high-frequency part of the superposition of different geological scales. The second part explains the derivation of scattering attenuation for low-frequency acoustic waves by $3 \mathrm{D}$ isotropic fractal heterogeneities using the mean field theory. Results comply with the Rayleigh regime and the Backus effective medium for very low frequency. We present analytical predictions of scattering attenuation and show the existence of a cutoff frequency for the penetration of waves. We use the frequency dependence of the penetration depth to calculate the shift of the dominant frequency of a Ricker wavelet. We conclude by suggesting further improvements.

\section{STATISTICAL MODEL OF HETEROGENEITIES}

Let us consider the spatial fluctuations of seismic velocities to be small and to constitute a second-order stochastic process. We describe the fluctuations by using different realizations of the random function $f(\mathbf{x})$ with the expectation value $\langle f\rangle=0$ and with the spatial covariance depending on the relative distance $\mathbf{r}$ defined by

$$
\langle f(\mathbf{x}) f(\mathbf{x}+\mathbf{r})\rangle=\sigma^{2} N(\mathbf{r})
$$

where $\sigma$ is the standard deviation and $N(\mathbf{r})$ is the spatial autocorrelation function with $N(\mathbf{0})=1$. The energy spectrum $E^{(s)}(\mathbf{k})$ of the fluctuations in $s$ dimensions $(s=1,2,3)$ is related to the autocorrelation by the Wiener-Khintchine theorem (Born and Wolf, 1964):

$$
\begin{aligned}
E^{(s)}(\mathbf{k})=|F(\mathbf{k})|^{2} & =\sigma^{2} \int N(\mathbf{r}) e^{-i \mathbf{k} \cdot \mathbf{r}} d \mathbf{r}, \\
F(\mathbf{k}) & =\int f(\mathbf{x}) e^{-i \mathbf{k} \cdot \mathbf{x}} d \mathbf{x}
\end{aligned}
$$

where $\mathbf{k}$ is the spatial wave vector and $F(\mathbf{k})$ is the Fourier transform of $f(\mathbf{x})$. The energy spectrum in equation 2 can be simplified, for an isotropic correlation function, to

$$
\begin{aligned}
& E^{(1)}(k)=2 \sigma^{2} \int_{0}^{\infty} N(r) \cos (k r) d r, \\
& E^{(3)}(k)=\frac{4 \pi}{k} \sigma^{2} \int_{0}^{\infty} r N(r) \sin (k r) d r,
\end{aligned}
$$

where $k=|\mathbf{k}|$. The von Kármán autocorrelation function $N_{H, b}(\mathbf{r})$ describes a selfaffine medium relevant for geological structures (Goff and Jordan, 1988; Holliger and Levander, 1992; Dolan et al., 1998; Sato and Fehler, 1998; Klimeš, 2002; Goff and Holliger, 2003). This function was initially derived by von Kármán (1948) while studying the velocity field in a turbulent fluid and has been used to describe heterogeneous media (Tatarski, 1961; Frankel and Clayton, 1986). The Fourier transform of $N_{H, b}(\mathbf{r})$ 
was given by Lord (1954). The statistical autocorrelation $N_{H, b}(\mathbf{r})$ and the energy spectrum $E_{H, b}^{(s)}(\mathbf{k})$ in the Fourier domain are

$$
\begin{aligned}
N_{H, b}(\mathbf{r})= & \frac{2^{(1-H)}}{\Gamma(H)}(r / b)^{H} K_{H}(r / b), \\
E_{H, b}^{(s)}(\mathbf{k})= & \sigma^{2} C_{H}^{(s)} \frac{(2 b)^{s}}{\left(1+b^{2} k^{2}\right)^{H+\frac{s}{2}}} \\
\text { with } & C_{H}^{(s)}=\left|\frac{\Gamma\left(H+\frac{s}{2}\right)}{\Gamma(H)}\right| \pi^{\frac{s}{2}}
\end{aligned}
$$

where $r=|\mathbf{r}|, K_{H}$ is the modified Bessel function of the second kind with order $H$, and $\Gamma$ is the Gamma function. Parameters describing the heterogeneities are characteristic distance $b$, below which the distribution is fractal, and exponent $H$, characterizing the roughness of the medium. We use the energy spectrum in equation 7 with $s=1$ to analyze sonic logs and with $s=3$ to predict 3D scattering attenuation.

\section{Fractal statistics}

Among different concepts introduced by the theory of fractals (Mandelbrot, 1983), self-affine property accounts for invariance of roughness of a curve observed at different scales. Self-affine fractals can be characterized by the power-law dependence of their energy spectrum $E(f)$ on frequency $f$ :

$$
E(f) \propto f^{-\beta}
$$

The exponent $\beta$, in the energy spectrum $E_{H, b}^{(s)}(\mathbf{k})$ from equation 7 , is

$$
\beta=2 H+s \text {. }
$$

For $\beta=0$, energy spectrum is constant and describes the familiar white noise. Causal integration of Gaussian white noise produces the classical Brownian motion, or random walk, characteristic of diffusion processes, and results in an energy spectrum with $\beta=2$. The autocorrelation function of Brownian motion signals is a decreasing exponential and the autocorrelation in equation 6 properly reduces to $\exp [-r / b]$ for $H=0.5$. Another interesting form of spectrum is for $\beta=1$. The associated signal is called Flicker noise (Schottky, 1926; Dolan et al., 1998) and can be interpreted as the superposition of different relaxation processes. For geological layers, such form of spectrum was interpreted as the expression of quasi-cyclicity and blocky layering (Shtatland, 1991). Generalization, including Gaussian white noise and Brownian motion, leads to two types of fractal signals (Shtatland, 1991; Turcotte, 1997; Li, 2003), namely

- fractional Gaussian noise (fGn) defined as filtered Gaussian white noise with $-1 \leq \beta \leq 1$, 
- fractional Brownian motion (fBm) built by causal integration of fGn above and resulting in $1 \leq \beta \leq 3$.

The fGn is stationary and Gaussian, whereas the fBm is neither stationary nor Gaussian. Exponent $\beta_{f B m}$ of the $\mathrm{fBm}$ is related to exponent $\beta_{f G n}$ of the fGn, used for integration, by $\beta_{f B m}=\beta_{f G n}+2$.

The significance of parameter $H$ in equation 10 is delicate and connected to the Hurst exponent $H u$, which measures the correlation of time series (Hurst, 1951) by

$$
H u=\frac{\log (R / \sqrt{S})}{\log (T)},
$$

where $R$ and $S$ are respectively range of variations and variance calculated for the length $T$ of the signal. The meaning of the value of $H u$ is

- antipersistence for $0 \leq H u \leq 0.5$,

- random process for $H u=0.5$, and

- persistence for $0.5 \leq H u \leq 1$.

Estimation of $H u$ using formula 11 is relevant only for fGn signals (Turcotte, 1997). For example, Gaussian white noise produces $H u=0.5$. Parameter $H$ defined in equation 10 is associated with the Hurst exponent by

- $H=H u-1$ for fGn with $-1 \leq \beta \leq 1$;

- $H$ equals the Hurst exponent of incremented fGn (Li, 2003) for fBm with $1 \leq$ $\beta \leq 3$.

The different self-affine 1D fractal models are presented in Table 1 according to the nature and persistency of the signal. A previous analysis of the logarithm of acoustic impedance from well data by Walden and Hosken (1985) shows $1 / 2 \leq \beta \leq 3 / 2$, promoting the so-called $1 / f$ geology. An improved solution, reproducing the complete well log sequence in sedimentary rocks, uses a similar random process based on fractional Lévy motion (Painter and Paterson, 1994), but fBm can be adequate at small scales, inside different facies (Lu et al., 2002).

\section{Synthetic realizations}

Our method of synthesizing correlated random media is summarized in Table 2 for fractional Gaussian noise (fGn) and fractional Brownian motion (fBm). The Gaussian nature of the initial white noise is contained in the phase of its Fourier transform $G(\mathbf{k})$, whereas the amplitude is constant with frequency because the noise is white. 


\begin{tabular}{|c|r|r|c|}
\hline Fractal exponent & Von Kármán exponent $H$ & Description & Geology \\
\hline$\beta=0$ & -0.5 & Gaussian white noise & Random process \\
\hline $0<\beta<1$ & $-0.5<H<0$ & Persistent fGn & \\
\hline$\beta=1$ & 0 & Flicker noise & Blocky layers \\
\hline $1<\beta<2$ & $0<H<0.5$ & Antipersistent fBm & Quasi-cyclic deposition \\
\hline$\beta=2$ & 0.5 & Brownian walk & Random deposition \\
\hline $2<\beta<3$ & $0.5<H<1$ & Persistent fBm & Transitional deposition \\
\hline
\end{tabular}

Table 1: Classification of 1D fractal statistics according to the exponent $\beta$ and geological interpretation.

\begin{tabular}{|c|l|c|c|}
\hline Steps & Domain & Operation for $f G n$ & Operation for $f B m$ \\
\hline 1 & Space & Generate Gaussian white noise $g(\mathbf{x})$ with zero mean and unit variance \\
\hline 2 & Fourier & \multicolumn{2}{|c|}{ Generate energy spectrum $E_{H, b}^{(s)}(\mathbf{k})$} \\
\hline 3 & Fourier & $F(\mathbf{k})=\sqrt{E_{H, b}^{(s)}(\mathbf{k})} G(\mathbf{k})$ & $F(\mathbf{k})=-i \operatorname{sign}(\mathbf{k}) \sqrt{E_{H, b}^{(s)}(\mathbf{k})} G(\mathbf{k})$ \\
\hline 4 & Space & Obtain correlated fGn $f(\mathbf{x})$ & Obtain correlated fBm $f(\mathbf{x})$ \\
\hline
\end{tabular}

Table 2: Synthesis of correlated heterogeneous media by generating fractional Gaussian noise (fGn) or fractional Brownian motion $(\mathrm{fBm})$ with the spectrum $E_{H, b}^{(s)}(\mathbf{k})$. The spatial Fourier transforms in $s$ dimensions of the distributions $f(\mathbf{x})$ and $g(\mathbf{x})$ are respectively $F(\mathbf{k})$ and $G(\mathbf{k})$ with $|G(\mathbf{k})|=1$, and $\operatorname{sign}(\mathbf{k})=\mathbf{k} /|\mathbf{k}|$. 


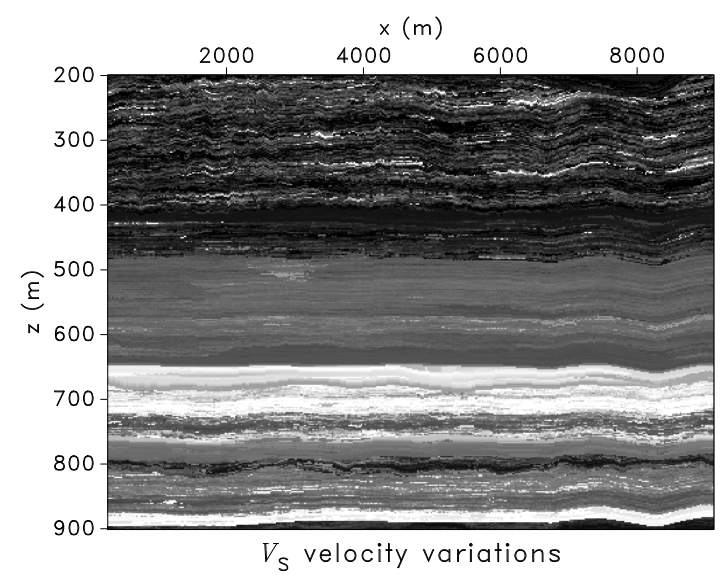

(a)

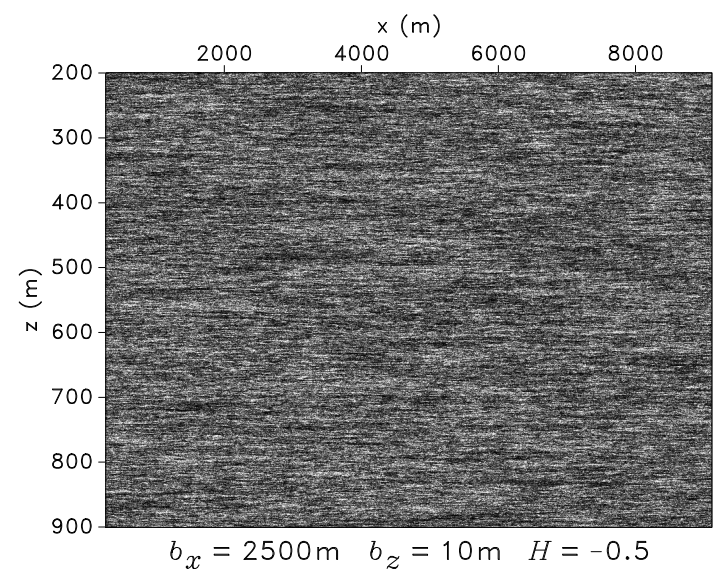

(c)

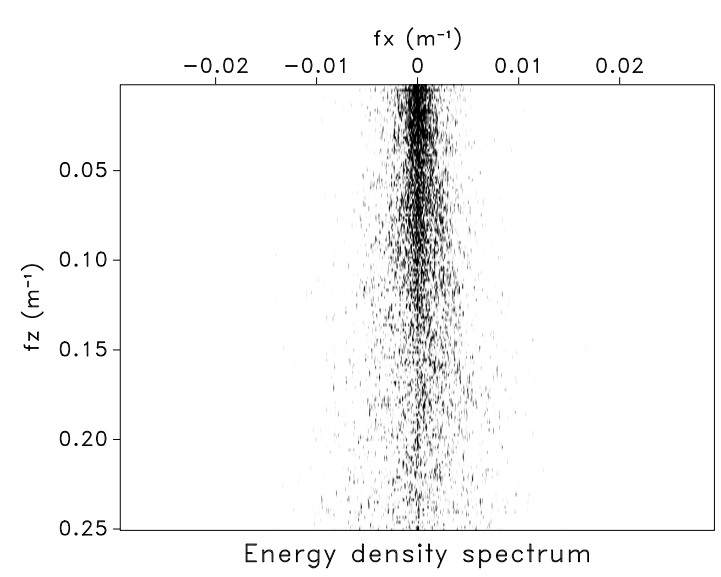

(b)

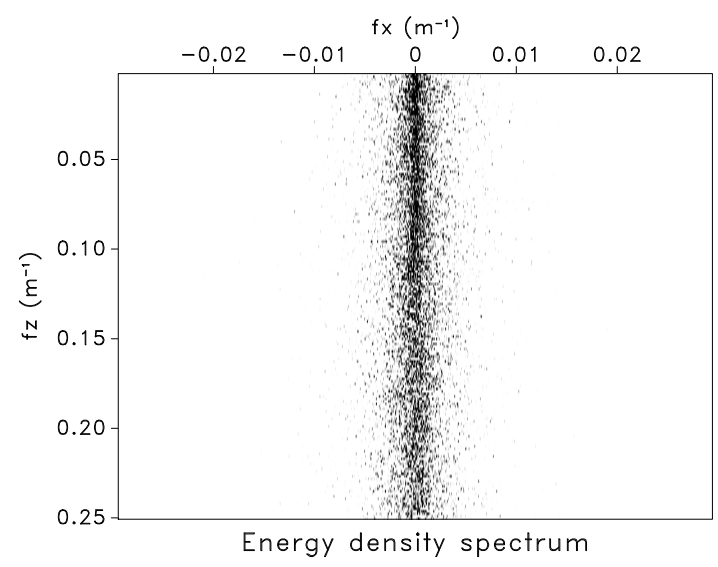

(d)

Figure 1: Variations of $V_{S}$ in a high-resolution reservoir model based on seismic and well data from a field in Canada (a) and its spectral energy density (b). Synthetic realization of $2 \mathrm{D}$ fGn using the von Kármán spectral amplitude with the exponent $\beta=1$ and elliptical anisotropy (c, d). 
The causal integration, to produce the fBm, is performed by a phase rotation in the Fourier domain, strictly equivalent to the Hilbert transform. The fractal property, below spatial scale $b$, is imposed by the amplitude $\sqrt{E_{H, b}^{(s)}}$ of the von Kármán model.

For dimension $s=2$, the exponent in equation 7 is $\beta=2 H+2$. Therefore, the energy spectrum of the $2 \mathrm{D}$ fGn with $H=-0.5$ in Figure 1 is $E(f) \propto 1 / f$. Klime (2002) in fact proposed using $-0.5 \leq H \leq 0$ to synthesize geologically realistic $2 \mathrm{D}$ models with the von Kármán function. Because sediments are made up of layers, we consider the autocorrelation function to be vertical transverse isotropic. We use two different correlation lengths $b_{x}$ and $b_{z}$ for horizontal and vertical directions, and define the Riemannian relative distance (Goff and Jordan, 1988):

$$
r / b=\sqrt{\left(r_{x} / b_{x}\right)^{2}+\left(r_{z} / b_{z}\right)^{2}}
$$

Figure 1 shows, for comparison, the signal and associated energy spectrum of the synthetic fGn and of a 2D section from a high-resolution model of a clastic reservoir in Canada. The spectrum of the synthetic heterogeneous medium is similar to the one from the reservoir model, but the synthetic fGn, although exhibiting some comparable roughness in the space domain, does not contain coherent, large geological structures, i.e. folded beds.

\section{Nonlinear parameter estimation on sonic well logs}

We propose to use the synthesis of a random medium detailed in Table 2 for $s=1$ as a basis for the procedure to estimate heterogeneity parameters from sonic logs. We achieve optimization by using a weighted least-squares method in the spectral domain on the logarithm of the amplitude, with the model derived from equation 7 :

$$
\begin{aligned}
\ln |F(k)| & =\ln |F(0)|-p \ln \left[1+(k b)^{2}\right], \\
p & =\frac{H}{2}+\frac{1}{4} \text { and } \\
|F(0)| & =\sigma \sqrt{2 b C_{H}^{(1)}} .
\end{aligned}
$$

We estimate the three parameters, $b, H$, and $\sigma$, using a separable least-squares method (Golub and Pereira, 1973) for $\ln |F(0)|$ and the slope $p$, and a Gauss Newton optimization algorithm on the nonlinear parameter $b^{2}$. Parameter $\ln |F(0)|$ is included in the optimization algorithm because it is difficult to estimate directly from the zerofrequency component in the data. Standard deviation $\sigma$, extracted from relation 15, is confirmed by direct evaluation on the spatial signal. When applying the method, we first substract the signal expectation and use it as a scaling factor. We have tested the efficiency of the algorithm on synthetic fGn and fBm generated by the procedure in Table 2 with a discrete length of 4056 points. Three synthetic fractal signals and their parameter estimations are shown in Figure 2. Results of the validation tests are presented in Table 3. 


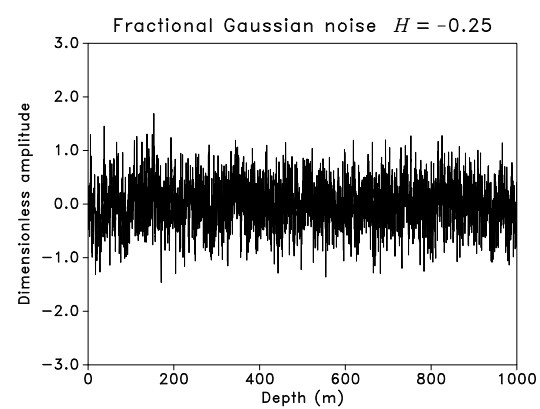

(a)

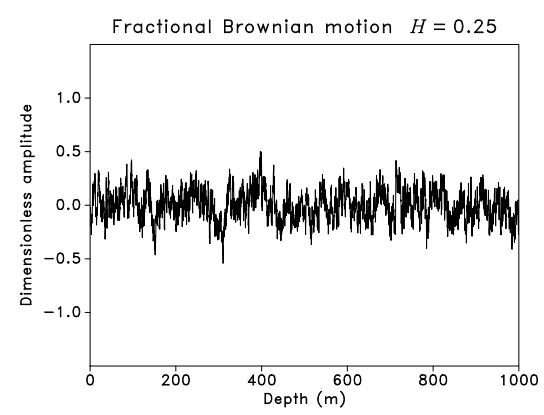

(c)

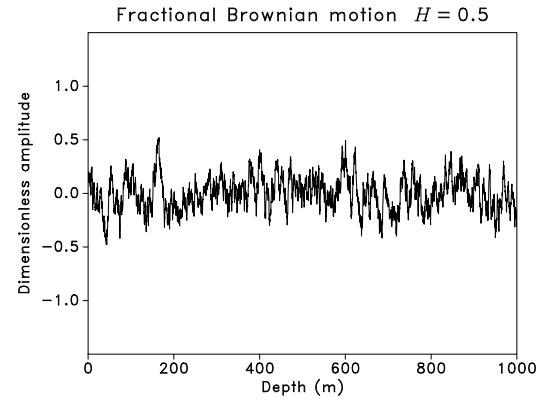

(e)

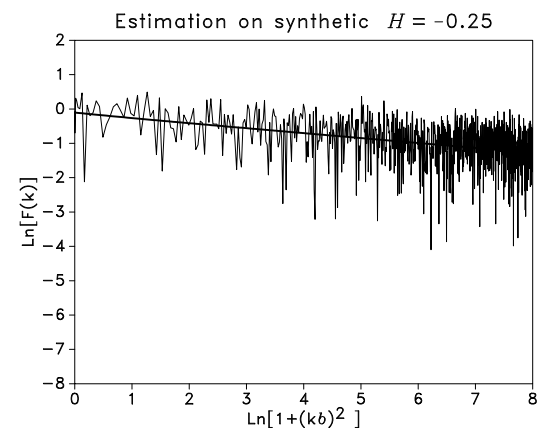

(b)

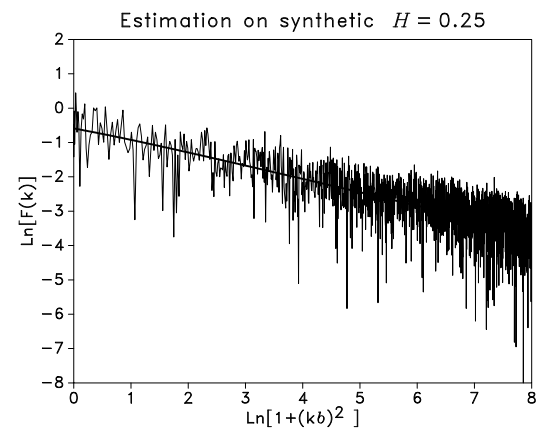

(d)

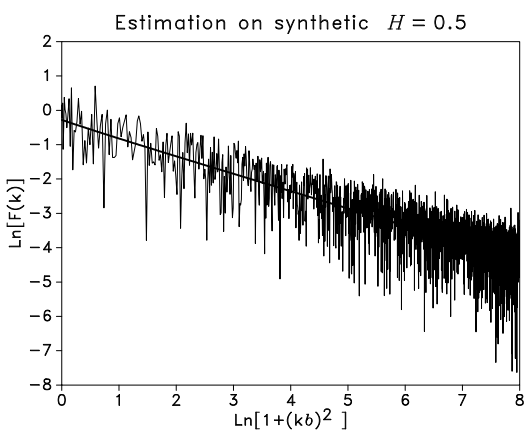

(f)

Figure 2: Synthetic signals generated as fGn with $H=-0.25, b=10 \mathrm{~m}, \sigma=20 \%$ in (a); fBm with $H=0.25, b=5 \mathrm{~m}, \sigma=30 \%$ in (c); and fBm with $H=0.5, b=5 \mathrm{~m}$, $\sigma=30 \%$ in (e). Parameter estimations on the logarithm of the spectral amplitude are shown on the right $(b, d, f)$. 


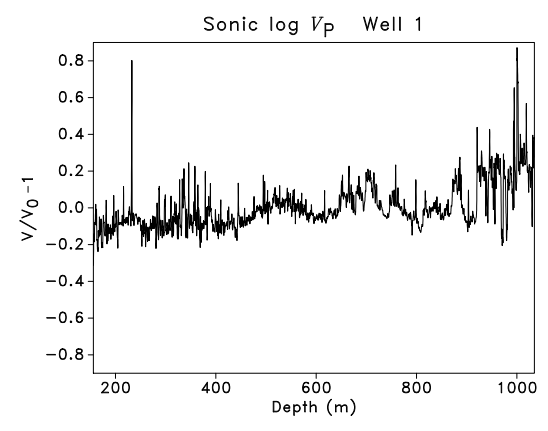

(a)

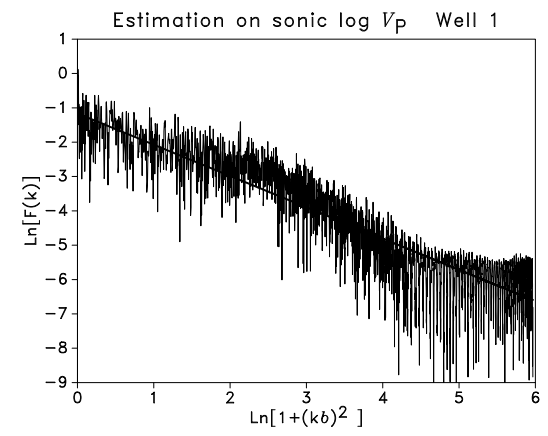

(c)

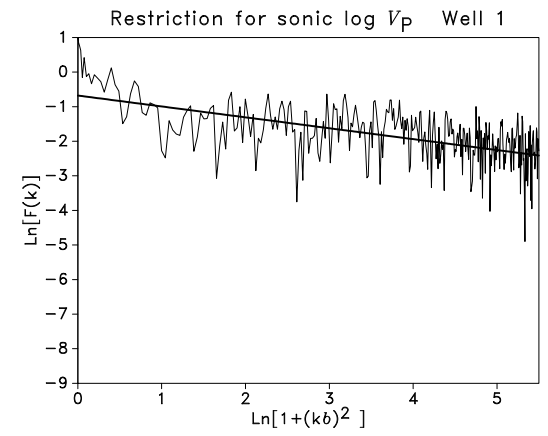

(e)

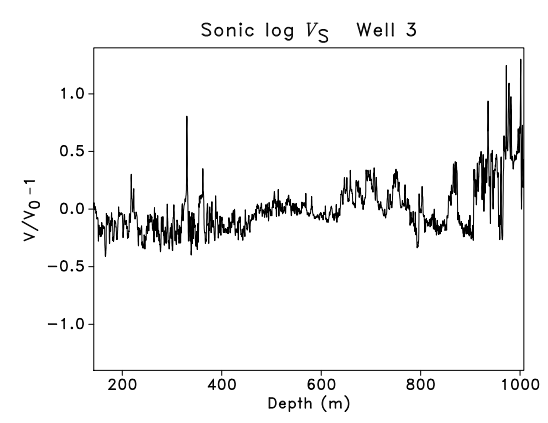

(b)

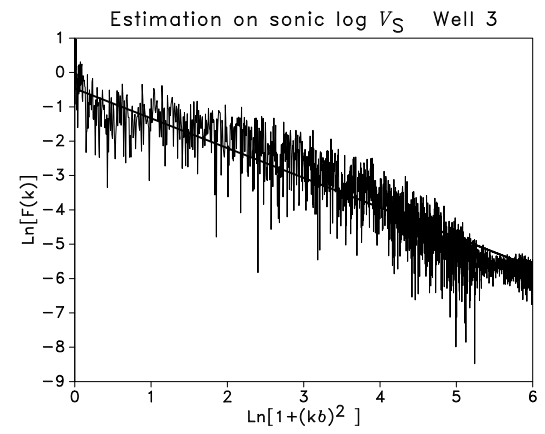

(d)

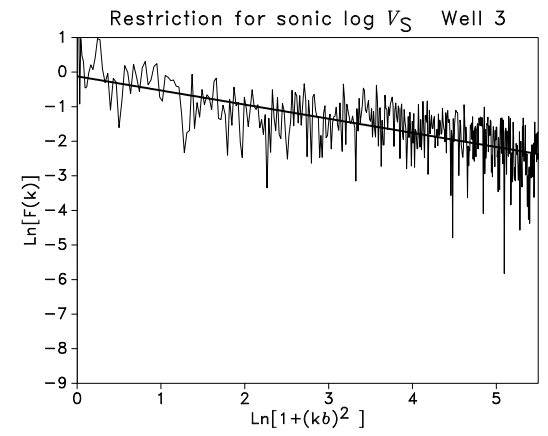

(f)

Figure 3: Sonic $\log V_{P}$ (a) from well $\mathrm{N}^{\circ} 1$ and $V_{S}$ (b) from well $\mathrm{N}^{\circ} 3$, scaled by their respective average value $V_{0}$. Parameter estimation on the logarithm of the spectral amplitude (c, d) shows the existence of different slopes for low, medium, and high frequencies. These tool artefacts are removed by restricting the estimation method to low frequency $(e, f)$. 


\begin{tabular}{|l|rcc|}
\hline Parameters & $H$ & $b(\mathrm{~m})$ & $\sigma(\%)$ \\
\hline Generated fGn & -0.25 & 10.0 & 20 \\
Recovered & -0.21 & 11.0 & 18 \\
\hline Generated fGn & -0.25 & 5.0 & 20 \\
Recovered & -0.21 & 5.9 & 17 \\
\hline Generated fBm & 0.25 & 10.0 & 30 \\
Recovered & 0.26 & 10.2 & 23 \\
\hline Generated fBm & 0.50 & 5.0 & 40 \\
Recovered & 0.51 & 5.3 & 32 \\
\hline Generated fBm & 0.75 & 3.0 & 40 \\
Recovered & 0.79 & 2.7 & 30 \\
\hline
\end{tabular}

Table 3: Comparison of the stochastic medium parameters used to generate synthetics fGn and $\mathrm{fBm}$ and their recovery by the nonlinear estimation method.

Well log data come from a sandy channel reservoir with a clastic overburden, and the facies evolves from silty sandstone to mudstone, which is characteristic of alluvial deposition. Velocities $V_{P}$ and $V_{S}$ were both measured with a spatial sampling of $0.125 \mathrm{~m}$. Figure 3 shows the parameter estimation for two sonic logs. Comparison with the method applied to the synthetics in Figure 2 uncovers the existence of different slopes for different frequencies in Figures 3(c) and 3(d). We can reasonably delimit three domains, denoted (A) for low frequencies, (B) for medium frequencies, and $(\mathrm{C})$ for very high frequencies. These domains can be identified by parameters $r_{S}$ and $r_{I}$, representing specific values of relative distance $r$, namely

$$
\text { (A) for } r \geq r_{S} \text {, (B) for } r_{S} \geq r \geq r_{I} \text {, (C) for } r_{I} \geq r \text {, }
$$

where $1 \mathrm{~m}<r_{S}<2 \mathrm{~m}$ and $r_{I}<1 \mathrm{~m}$. The sharp break (B) in the medium frequencies followed by a white noise (C) at high frequencies is characteristic of the tool artefact. Data acquisition involves a convolution with a box-car window (Shiomi et al., 1997; Dolan et al., 1998). Application of the estimation method is thus restricted to relative distances $r>r_{S}$, and results are shown in Figures 3(e) and 3(f).

Results are summarized in Table 4 for the four different sonic $\operatorname{logs} V_{P}$ and $V_{S}$. The ratio $\left\langle V_{P}\right\rangle /\left\langle V_{S}\right\rangle$ is almost constant for the four well logs and roughly equal to two. The updated estimation in the spatial wavelength domain (A) produces reasonable results in Table 4. Standard deviation $\sigma$ varies from 20 to $45 \%$ and is larger for $V_{S}$ $\operatorname{logs}$ than for $V_{P} \operatorname{logs}$. Correlation length $b$ is about $5 \mathrm{~m}$ for both $V_{P}$ and $V_{S}$, except for the well $\mathrm{N}^{\circ} 4$, which is $2.5 \mathrm{~m}$. Exponent $H$ for $V_{P}$ varies from 0.1 to 0.4 and for $V_{S}$ from 0.2 to 0.6. In Figure 4, comparison of the frequency content of one real sonic log with one realization of a synthetic $\mathrm{fBm}$, generated using similar parameters, shows that the sonic-log data contain higher peaks for very large wavelengths. We detected in the different sonic well logs the recurrence of some particular spatial cycles at $2.5 \mathrm{~m}$, $5 \mathrm{~m}, 10 \mathrm{~m}$, and $20 \mathrm{~m}$. 


\begin{tabular}{|c|c|c|c|c|}
\hline Well & $b(\mathrm{~m})$ & $H$ & $\sigma(\%)$ & $\langle V\rangle(\mathrm{m} / \mathrm{s})$ \\
\hline \multirow{4}{*}{$\begin{array}{rr}\mathrm{N}^{\circ} 1 & V_{P} \text { full } \\
& r>1.5 \mathrm{~m} \\
& V_{S} \text { full } \\
r>2.0 \mathrm{~m}\end{array}$} & 0.79 & 1.32 & 17 & \multirow[t]{2}{*}{$\underline{2791}$} \\
\hline & $\underline{6.70}$ & $\underline{0.13}$ & $\underline{22}$ & \\
\hline & 1.05 & 1.16 & 32 & \multirow[t]{2}{*}{$\underline{1218}$} \\
\hline & $\underline{5.92}$ & $\underline{0.21}$ & $\underline{35}$ & \\
\hline \multirow{4}{*}{$\begin{array}{r}\mathrm{N}^{\circ} 2 \quad V_{P} \text { full } \\
r>1.6 \mathrm{~m} \\
V_{S} \text { full } \\
r>1.5 \mathrm{~m}\end{array}$} & 1.90 & 0.92 & 27 & \multirow[t]{2}{*}{$\underline{2842}$} \\
\hline & $\underline{5.34}$ & $\underline{0.38}$ & $\underline{29}$ & \\
\hline & 2.84 & 0.83 & 45 & \multirow[t]{2}{*}{$\underline{1240}$} \\
\hline & $\underline{3.08}$ & $\underline{0.62}$ & $\underline{44}$ & \\
\hline \multirow{4}{*}{ 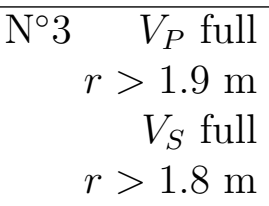 } & 1.34 & 1.16 & 20 & \multirow[t]{2}{*}{$\underline{2787}$} \\
\hline & $\underline{7.22}$ & $\underline{0.18}$ & $\underline{21}$ & \\
\hline & 1.25 & 1.23 & 32 & \multirow[t]{2}{*}{$\underline{1216}$} \\
\hline & $\underline{5.01}$ & $\underline{0.32}$ & $\underline{36}$ & \\
\hline \multirow{4}{*}{ 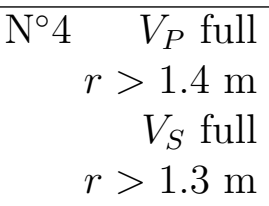 } & 0.64 & 1.98 & 18 & \multirow[t]{2}{*}{2745} \\
\hline & $\underline{2.58}$ & $\underline{0.39}$ & $\underline{38}$ & \\
\hline & 0.57 & 2.26 & 32 & \multirow[t]{2}{*}{$\underline{1247}$} \\
\hline & $\underline{2.46}$ & $\underline{0.56}$ & $\underline{33}$ & \\
\hline
\end{tabular}

Table 4: Parameters estimation from four wells in a clastic overburden, for the full sonic logs, and for limited spatial frequency bandwidths using the indicated restriction on the relative distance $r$ to remove the tool artefacts. Relevant physical values are underlined.

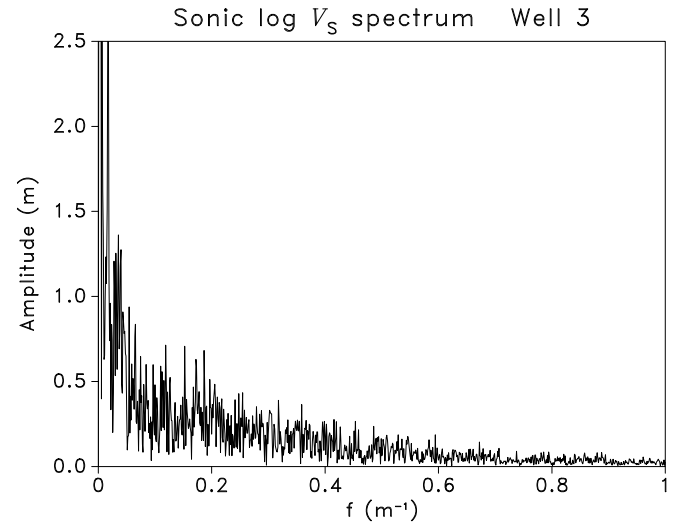

(a)

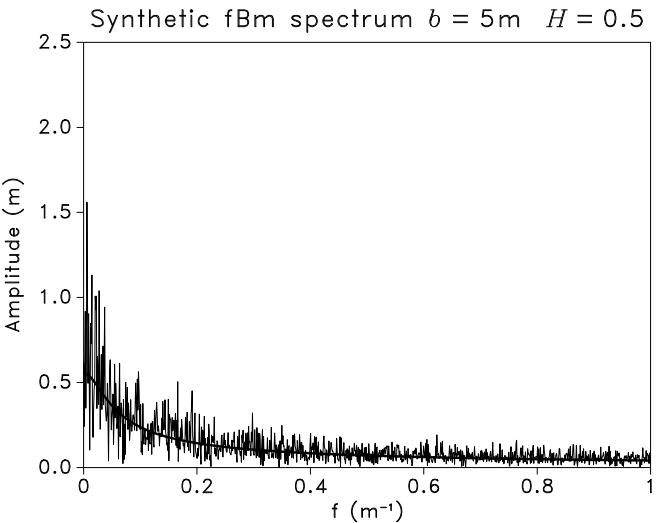

(b)

Figure 4: Fourier spectrum of the scaled $V_{S}$ sonic log from well $\mathrm{N}^{\circ} 3$ (a). The shape of the low-frequency content is different from that of the Fourier spectrum of the fractional Brownian motion (b) synthesized with the von Kármán model using $H=0.25, b=5 \mathrm{~m}$, and $\sigma=30 \%$. 


\section{Fractal heterogeneities and cycles in sediments}

O'Doherty and Anstey (1971) and Anstey and O'Doherty (2002a) described variations in well logs by the superposition of different types of deposition, leading to "layers inside layers". Their classification includes

1. A large number of small thickness layers $(\leq 1 \mathrm{~m})$ for weakly transitional depositions with small reflection coefficients;

2. Cyclic layers of thicknesses from 1 to $10 \mathrm{~m}$ with sharp interfaces, corresponding to fine layering depositions inside a facies for short-period sea cycles; and

3. Horizons imaged by seismic reflection, i.e. different facies for a small number of thicker blocky layers associated with low-order cycles.

They suggested that transmission losses could be compensated by multiple reflections, depending on seismic wavelength. This classification is in agreement with the fact that high exponents, $H$, appear for shorter scales, $b$, in Table 4 . The estimation performed on the sonic logs indicates fractal properties for distances shorter than $b \simeq 5 \mathrm{~m}$. Acccording to Anstey and O'Doherty (2002a), well log signals are the superposition of several processes with different scales. The von Kármán model captures part of it. Parameters extracted by our analysis describe heterogeneities corresponding to type 2 of the O'Doherty-Anstey classification, which is a fractal behavior inside major geological units, at least from 10 down to $1 \mathrm{~m}$, with a correlation length of $5 \mathrm{~m}$. Previous estimations of the correlation length on well logs were produced by direct calculation of the spatial autocorrelation (White et al., 1990; Shiomi et al., 1997). White et al. (1990) suggested the possibility of superposition of two correlation lengths at 5 and $20 \mathrm{~m}$. The wavelet detection analysis of gamma-ray and resistivity well logs for a sandstone confirmed the strong evidence of local cyclicity in the stratigraphic sequences (Rivera et al., 2004). We think that direct estimation of correlation distance $b$ using the autocorrelation function, or our estimation method, captures the shortest dominant cycle in the sedimentary layers. This would explain why the fractal behavior seems to hold for larger scales in Figures 3(e) and 3(f).

Parameter $H$, estimated from well $\operatorname{logs}$, is $0<H \leq 0.5$ and consistent with an antipersistent fractional Brownian motion characteristic of cyclicity (see Table 1). The Hurst exponent commonly exhibits some antipersistence in sediments with values from 0.2 to 0.5 for sandstones (Dolan et al., 1998; Lu et al., 2002). High values of 0.5 and 0.6 could be interpreted, in a clastic context, to be caused by a transitional deposition involving persistency, as in natural floods. Natural flood records exhibit a Hurst exponent, $0.5 \leq H u \leq 1.0$, associated with so-called black noise (Hurst, 1951; Mandelbrot and Wallis, 1969). 


\section{Seismic-scale heterogeneities}

Inside a main facies, we can not recover the short wavelengths of type 1 in the O'Doherty-Anstey classification because of well-logging tool limitation. Type 3 of the classification includes Milankovitch cycles of about 10 to $20 \mathrm{~m}$ and third-order sea-level cycles from 15 to $300 \mathrm{~m}$ (Anstey and O'Doherty, 2002a). Seismic reflectors are conventionally identified as being chronostratigraphic horizons separating different geological units. They correspond to the wavelength of conventional seismic surveys, up to $100 \mathrm{~Hz}$, and should induce some resonant scattering with "friendly" multiples. Multipathing is observed for this ratio of seismic wavelength to the size of heterogeneities, as shown by numerical experiments (Frankel and Clayton, 1986). This domain should be treated using wave-localization theory and the Rytov method. These could nevertheless fail to explain the data because of quasi-periodicity of the medium at this scale, violating the ergodicity assumption required by wave-localization theory. Statistical methods using the autocorrelation function seem to be adapted to describe quasi-periodic media when the ratio of $b$ over the seismic wavelength is small. When the wavelength is of the same size, local quasi-cyclicity of the sedimentary sequence should not be ignored (Morlet et al., 1982; Stovas and Ursin, 2007).

The non-Gaussian nature and non-stationarity of sedimentary layers call for more sophisticated methods to be used, especially in order to capture larger scale pseudocyclic heterogeneities, as, for example, a multifractal analysis (Marsan and Bean, 2003) or a local cyclicity detection by wavelet analysis (Rivera et al., 2004). The wavelet transform was indeed introduced so that seismic signals in locally cyclic sedimentary layers could be analyzed (Morlet et al., 1982).

\section{SCATTERING ATTENUATION IN 3D}

Different scattering regimes exist when waves propagate in heterogeneous media, according to the ratio of the wavelength, $\lambda$, to the size, $b$, of heterogeneities. The formalism including the different scattering regimes, when heterogeneities are modeled by spherical inclusions, is the Mie scattering theory. Recent experimental results (Le Gonidec and Gibert, 2007) on sonic-wave reflectivity in a granular medium, made up of beads of size $b$ in a water tank, illustrate this classification :

1. for low frequencies, when $\lambda>\pi b$, backward scattering is dominant, the Born approximation can be used, and the regime is Rayleigh scattering;

2. for wavelengths similar in size to heterogeneity, when $\pi b>\lambda>\pi b / 2$, lateral scattering is important, multiples should not be neglected, and the regime is called resonant scattering; and

3. for high frequencies, when $\pi b / 2>\lambda$, waves are scattered mainly forward, and localization theory and the Rytov formalism are appropriate. 
Experiments have demonstrated that wave reflectivity strongly decreases when the wavelength of the incident wave is twice the diameter of the beads, for which lateral scattering starts to be dominant. Mean wavefield formalism is valid only for a low frequency, when the wavelength is larger than the size of heterogeneities because of the assumptions in the derivation (Karal and Keller, 1964). The Born approximation can describe the Rayleigh regime and the approach of resonant phase scattering (Frankel and Clayton, 1986; Sato and Fehler, 1998). For wavelengths shorter than the size of heterogeneities, artificial decoherence by phase randomization occurs (Wu, 1982; Sato and Fehler, 1998). We intend to describe the 3D attenuation in a stochastic fractal medium, when $k b \leq 1$, which is relevant for seismic survey frequencies. The limit of validity corresponds to wavelengths approaching the size of heterogeneities. We assume heterogeneities to be isotropic. The schemes in Figure 5 compare a realistic geological structure with two different end-member models. Scattering calculations in $1 \mathrm{D}$ underestimate the scattering loss by small-scale heterogeneities.

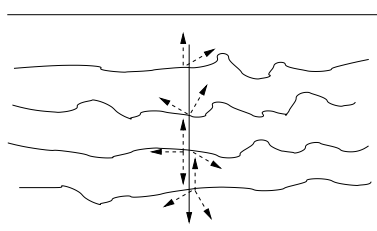

(a)

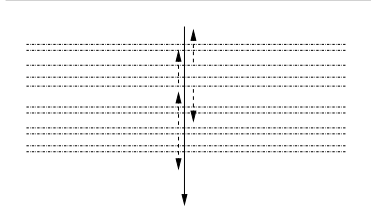

(b)

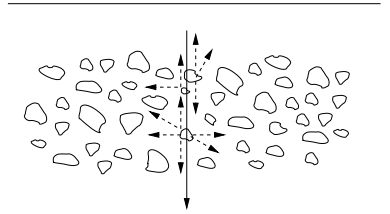

(c)

Figure 5: Schematic comparison of single scattering effects, during a vertical wave propagation in sediments, between a realistic geological structure (a) and two endmember models: horizontal layers with propagation including 1D scattering (b) and isotropic heterogeneities with $3 \mathrm{D}$ scattering (c).

\section{Low-frequency waves in $3 \mathrm{D}$ isotropic heterogeneous media}

A scalar wave $u(\mathbf{x}, \omega)$ in a weakly inhomogeneous medium (Chernov, 1960; Tatarski, 1961; Karal and Keller, 1964) satisfies the Helmholtz wave equation

$$
\Delta u(\mathbf{x}, \omega)+k_{0}^{2}[1+f(\mathbf{x})]^{2} u(\mathbf{x}, \omega)=0,
$$

where $f(\mathbf{x})$ is a small perturbation of the medium from homogeneity, and $k_{0}=\omega / c_{0}$. Phase velocity $c_{0}$ is the background velocity. Assuming a second-order stationary statistical distribution for fluctuations $f(\mathbf{x})$ and a zero expectation value $\langle f\rangle=0$, spatial covariance of the velocity variations is defined by relation 1 . Expectation $\langle u(\mathbf{x}, \omega)\rangle$ of random plane-wave realizations is calculated (Karal and Keller, 1964) using a perturbation theory to the second order in $f(\mathbf{x})$ by

$$
\left[\Delta+k_{0}^{2}\left(1+\sigma^{2}\right)\right]\langle u(\mathbf{x}, \omega)\rangle-4 k_{0}^{4} \sigma^{2} \int N\left(\mathbf{x}^{\prime}-\mathbf{x}\right) G\left(\mathbf{x}, \mathbf{x}^{\prime}, \omega\right)\left\langle u\left(\mathbf{x}^{\prime}, \omega\right)\right\rangle d \mathbf{x}^{\prime}=0
$$

where $G\left(\mathbf{x}, \mathbf{x}^{\prime}, \omega\right)$ is Green's function of the operator $\left[\Delta+k_{0}^{2}\right]$, and integration is performed over the $3 \mathrm{D}$ space. The dispersion relation for a plane wave propagating 
in the heterogeneous medium follows as

$$
\begin{aligned}
\frac{k^{2}}{k_{0}^{2}} & =1+\sigma^{2}\left[1-4 k_{0}^{2} \int N(\mathbf{r}) G(\mathbf{r}, \omega) e^{i \mathbf{k} \cdot \mathbf{r}} d \mathbf{r}\right], \\
G(\mathbf{r}, \omega) & =\frac{e^{i k_{0} r}}{4 \pi r}
\end{aligned}
$$

where $\mathbf{r}=\mathbf{x}^{\prime}-\mathbf{x}$ is the relative position, with an absolute value $r=|\mathbf{r}|$, and $G(\mathbf{r}, \omega)$ is the 3D isotropic free-space Green's function with the outward radiation condition (Bleistein et al., 2001). The path of waves should be sufficiently long to significantly sample medium heterogeneities statistically (Gist, 1994). The Born approximation is present because of Green's function. Heterogeneities with the isotropic correlation function $N(r)$ produce an isotropic wave vector $\mathbf{k}$. Combining Green's function in equation 19 with the isotropic integral in equation 5 reduces the squared dispersion relation of equation 18 to

$$
\frac{k^{2}}{k_{0}^{2}}=1+\sigma^{2}\left[1-\frac{4 k_{0}^{2}}{k} \int_{0}^{\infty} N(r) e^{i k_{0} r} \sin (k r) d r\right] .
$$

Second-order expansion $k / k_{0}=1+O\left(\sigma^{2}\right)$ in the solution constrains validity to the domain $k_{0} b \ll 1 / \sigma$, where $b$ is the characteristic length scale of the heterogeneities. The second-order approximation for the $3 \mathrm{D}$ dispersion relation is finally

$$
\frac{k}{k_{0}}=1+\frac{\sigma^{2}}{2}+i \sigma^{2} k_{0}\left[S(0)-S\left(2 k_{0}\right)\right] .
$$

Quantity $S(k)$, introduced above, is related to the real and even function $E^{(1)}(k)$, defined by the isotropic integral of equation 4 :

$$
\begin{aligned}
S(k) & =\int_{0}^{+\infty} N(r) e^{i k r} d r, \\
E^{(1)}(k) \sigma^{-2} & =\int_{-\infty}^{+\infty} N(r) e^{i k r} d r=2 \operatorname{Re}[S(k)], \\
2 i \operatorname{Im}[S(k)] & =S(k)-S(-k) .
\end{aligned}
$$

Connection to the O'Doherty-Anstey formula is detailed in Appendix A.

\section{Attenuation in 3D fractal media}

The energy spectrum, $E_{H, b}^{(1)}(k)$, of von Kármán's autocorrelation function $N_{H, b}(r)$ in equation 7 is real and even:

$$
\begin{aligned}
E_{H, b}^{(1)}(k) \sigma^{-2} & =\int_{-\infty}^{+\infty} N_{H, b}(r) e^{i k r} d r \\
& =C_{H}^{(1)} \frac{2 b}{\left(1+b^{2} k^{2}\right)^{H+\frac{1}{2}}}
\end{aligned}
$$


Values of $S(k)$ defined by equation 22 are

$$
\begin{aligned}
S(0) & =C_{H}^{(1)} b \\
\operatorname{Re}\left[S\left(2 k_{0}\right)\right] & =\frac{C_{H}^{(1)} b}{\left(1+4 b^{2} k_{0}^{2}\right)^{H+\frac{1}{2}}} .
\end{aligned}
$$

Coefficient $C_{H}^{(1)}$, defined by equation 8 , is an increasing function of exponent $H$ and has to be calculated numerically, except for some specific values:

$$
\begin{array}{lll}
C_{H}^{(1)} \sim \pi / \Gamma(H) \rightarrow 0 & \text { for } H \rightarrow 0, & \\
C_{-0.25}^{(1)}=1.3110 \ldots, & C_{0.25}^{(1)}=0.5991 \ldots, & C_{0.5}^{(1)}=1, \\
& C_{0.75}^{(1)}=1.3317 \ldots, & C_{1}^{(1)}=\pi / 2 .
\end{array}
$$

The dispersion relation of equation 21 solves for an explicit solution of attenuation and dispersion :

$$
\begin{aligned}
\operatorname{Re}\left[k / k_{0}\right] & =1+\frac{\sigma^{2}}{2}\left(1+2 k_{0} \operatorname{Im}\left[S\left(2 k_{0}\right)\right]\right), \\
\operatorname{Im}\left[k / k_{0}\right] & =\sigma^{2} k_{0} b C_{H}^{(1)}\left[1-\frac{1}{\left(1+4 b^{2} k_{0}^{2}\right)^{H+\frac{1}{2}}}\right] .
\end{aligned}
$$

When $H=0.5$, the derivation produces simple expressions as detailed in Appendix B. The use of $S^{*}(k)=S(-k)$ with the Kramers-Krönig relation can be used to determine the real part of $k$. In the context of the second-order approximation, scattering attenuation in a von Kármán isotropic medium is

$$
\frac{1}{Q}=\frac{2 \operatorname{Im}[k]}{\operatorname{Re}[k]}=2 \sigma^{2} k_{0} b C_{H}^{(1)}\left[1-\frac{1}{\left(1+4 b^{2} k_{0}^{2}\right)^{H+\frac{1}{2}}}\right] .
$$

For $k_{0} b \ll 1$, the scattering attenuation reduces to the Rayleigh diffusion regime:

$$
\frac{1}{Q} \simeq 8 \sigma^{2} C_{H}^{(1)}\left(H+\frac{1}{2}\right)\left(k_{0} b\right)^{3} .
$$

\section{Penetration depth}

Waves propagating in disordered media are exponentially attenuated by scattering (O'Doherty and Anstey, 1971; White et al., 1990). We define penetration depth $d(f)$ to be the skin depth (van der Baan et al., 2007) for low-frequency waves propagating in the heterogeneous medium:

$$
\begin{aligned}
\frac{1}{d(f)} & =\frac{k_{0}}{2 Q}=\operatorname{Im}[k], \\
d(f) & =\frac{b\left(1+4 b^{2} k_{0}^{2}\right)^{H+\frac{1}{2}}}{\sigma^{2}\left(k_{0} b\right)^{2} C_{H}^{(1)}\left[\left(1+4 b^{2} k_{0}^{2}\right)^{H+\frac{1}{2}}-1\right]},
\end{aligned}
$$


where $f$ is frequency. Penetration depth $d(f)$ corresponds to a decrease of wave amplitude by $1 / e$. For two-way traveltime, recorded amplitude is $14 \%$ of the initial one. Figure 6 shows the frequency dependence of the penetration depth for different values of parameters $b$ and $H$ and the scattering attenuation for acoustic P- and Swaves. Seismic background velocities are $V_{P}=2700 \mathrm{~m} / \mathrm{s}$ and $V_{S}=1230 \mathrm{~m} / \mathrm{s}$.

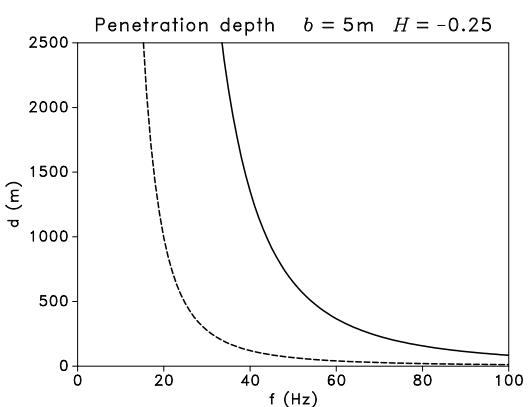

(a)

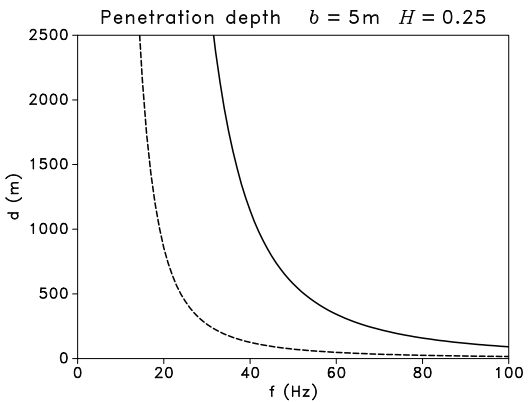

(c)

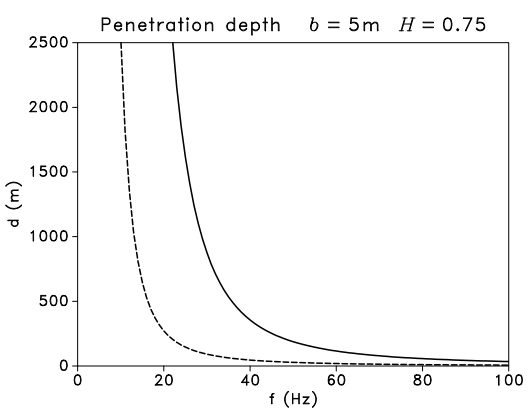

(e)

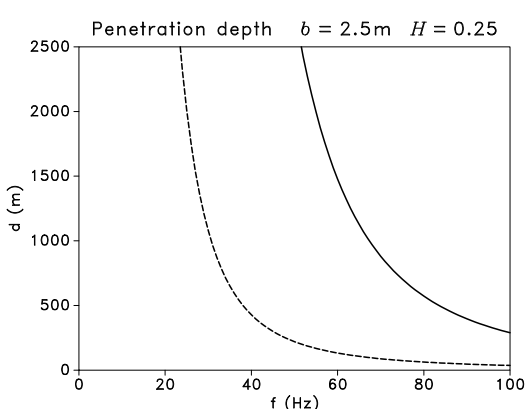

(b)

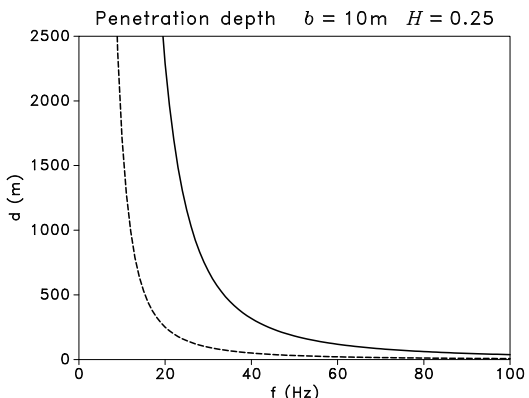

(d)

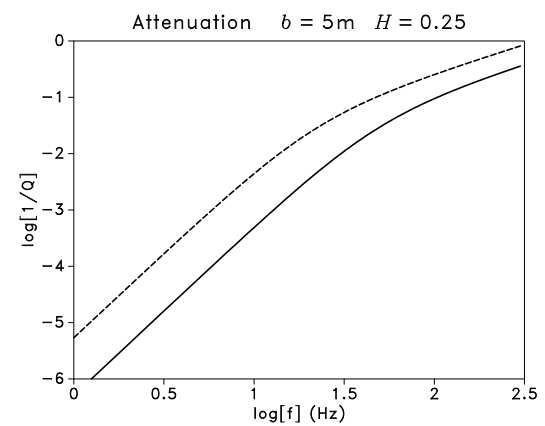

(f)

Figure 6: Penetration depth for P (solid) and S (dashed) scalar waves in heterogeneous media described by the von Kármán model with $\sigma=30 \%$. For $b=5 \mathrm{~m}$, a higher exponent $H$ decreases the penetration (a, c,e). The value of $b$ strongly influences the penetration of waves $(b, d)$. The slope break in the attenuation curve (f) determines the frequency below which our scattering model is valid.

Scattering attenuation $1 / Q$ is proportional to $1 / \lambda^{3}$ at low frequencies and corresponds to the Rayleigh diffusion regime. Attenuation increases at higher frequencies, where $1 / Q$ is proportional to $1 / \lambda$ and wavelength is comparable to the size of the heterogeneities. Nevertheless, the validity of our scattering theory is constrained to 
the low-frequency bandwidth until the attenuation curves in Figure 6(f) reach the change of slope at $45 \mathrm{~Hz}$ for S-waves and $70 \mathrm{~Hz}$ for P-waves. For a conventional seismic survey and for correlation length $b=5 \mathrm{~m}$, previously estimated, wavelengths of $\mathrm{P}$ - and S-waves and ratio $\lambda / b$ are indicated in Table 5.

\begin{tabular}{|c|c|c|c|}
\hline Wave & Frequency $(\mathrm{Hz})$ & $\lambda(\mathrm{m})$ & $\lambda / b$ \\
\hline $\mathrm{P}$ & 10 & 270 & 54 \\
& 90 & 30 & 6 \\
\hline $\mathrm{S}$ & 10 & 123 & 24 \\
& 50 & 25 & 5 \\
\hline
\end{tabular}

Table 5: Ratio of the wavelength $\lambda$ over the size of heterogeneities $b=5 \mathrm{~m}$ for realistic seismic frequencies, when $V_{P}=2700 \mathrm{~m} / \mathrm{s}, V_{S}=1230 \mathrm{~m} / \mathrm{s}$.

Scattering is more important for seismic wavelengths with a dimension similar to that of heterogeneities: high frequencies and S-waves, because their wavelengths are shorter than P-waves, are more attenuated. Penetration depth is close to infinity at very low frequencies but decreases drastically in a narrow frequency band, depending on parameters $H$ and $b$ (see Figure 6). This steep descent shifts to higher frequencies when the fractal exponent decreases, corresponding to a stronger cyclicity of the layers. A shorter correlation length of heterogeneities highly improves penetration of high frequencies for both types of wave (see Figures 6(b) and 6(d)). For large-size heterogeneities, i.e. $b>20 \mathrm{~m}$, the scattering theory we use is not valid because the seismic frequencies statisfy $k_{0} b \geq 1$. Scattering regimes and the suggested description of heterogeneities are summarized in Figure 7. Our results therefore ignore the effects of large cycles in sediments. We refer the reader to Stovas and Ursin (2007) for more information on the effects of cycles on wave progagation.

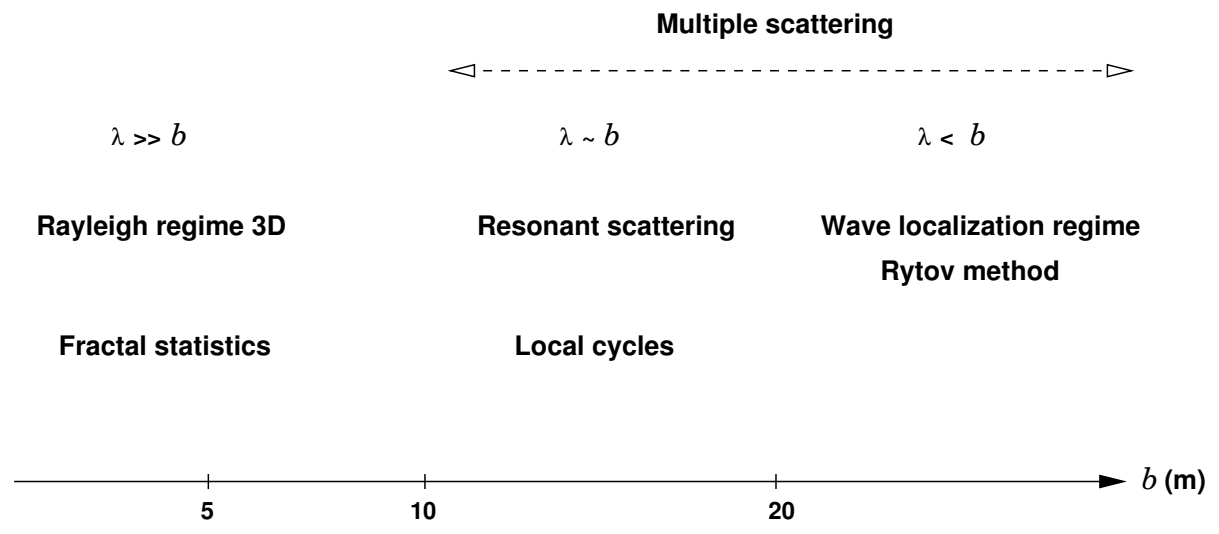

Figure 7: Schematic representation, including the suggested description of heterogeneities, of the different scattering regimes depending on the ratio between the seismic wavelength $\lambda$ and the size of heterogeneities $b$. The scale is for indication only and depends on the frequency band of the survey. 
Dominant frequency versus depth

If seismic pulse is defined as a Ricker wavelet, a relation can be derived for modification of the frequency content of $\mathrm{P}$ and $\mathrm{S}$ acoustic waves by scattering attenuation. Dominant frequency $f_{\text {dom }}(z)$ with depth $z$ and initial spectrum $S_{I}(f)$ of the source are defined by

$$
\begin{aligned}
S_{I}(f) & =\left(\frac{f}{f_{0}}\right)^{2} e^{-f^{2} / f_{0}^{2}}, \\
f_{\text {dom }}(z) & =\frac{d}{d f}\left(S_{I}(f) e^{-z / d(f)}\right),
\end{aligned}
$$

with initial condition $f_{\text {dom }}(0)=f_{0}$, and where $d(f)$ is the penetration depth defined in equation 34. Dispersion involves different traveltimes at different frequencies but does not modify the frequency content or amplitude. For convenience, we estimate dominant frequency as frequency expectation:

$$
\begin{aligned}
f_{\text {dom }}(z) & =\frac{1}{\left\langle S_{I}\right\rangle_{z}} \int f S_{I}(f) e^{-z / d(f)} d f, \\
\left\langle S_{I}\right\rangle_{z} & =\int S_{I}(f) e^{-z / d(f)} d f .
\end{aligned}
$$

Figure 8 shows the evolution of the dominant frequency with depth in fractal media, with $V_{P}=2700 \mathrm{~m} / \mathrm{s}, V_{S}=1230 \mathrm{~m} / \mathrm{s}$, and standard deviation $\sigma=30 \%$. The value of correlation length $b$ again has a very high impact, whereas the fractal exponent moderately influences results. For a multicomponent seismic survey in a clastic reservoir, evolution of the peak frequency should show a more important decrease with depth for PS data than for PP data.

\section{DISCUSSION}

The attenuation caused by anticorrelated 3D small-scale heterogeneities can be explained by a low-frequency scattering theory. The length scales that we estimated from sonic logs justify this approach for conventional seismic frequencies. The intensity of scattering attenuation and the value of frequency cutoff strongly depend on the size of the heterogeneities, and S-waves are more attenuated than $\mathrm{P}$-waves at the same frequency. Using low-frequency P-waves provides a better depth of penetration. More reflectors can be detected and imaged, but, of course, with less resolution. This phenomenon was observed in sub-basalt imaging (Ziolkowski et al., 2003). Our analysis of sonic logs confirms the relevance of a fractal description for the high-frequency content of quasi-periodic geological layers. Because sediments are highly stratified, their layered structure has previously motivated use of $1 \mathrm{D}$ models for seismic scattering attenuation, but a realistic estimate needs to be conducted in 3D. More generally, description of geological heterogeneities and use of scattering theories should be as depicted in Figure 7. 


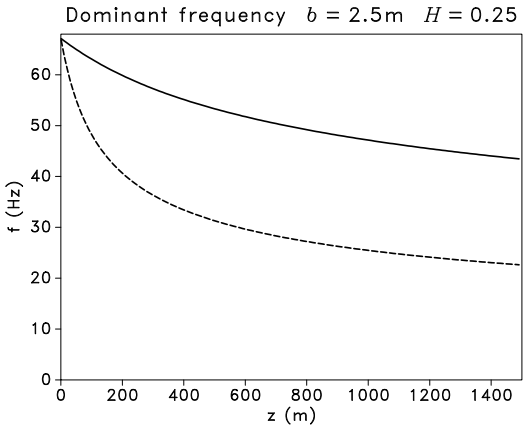

(a)

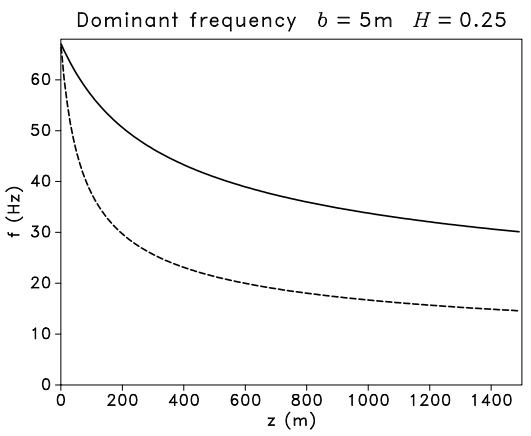

(c)

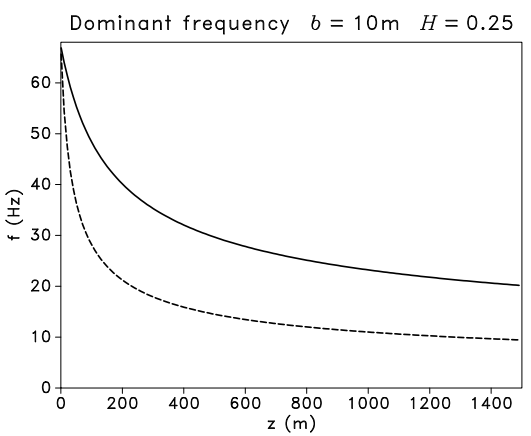

(e)

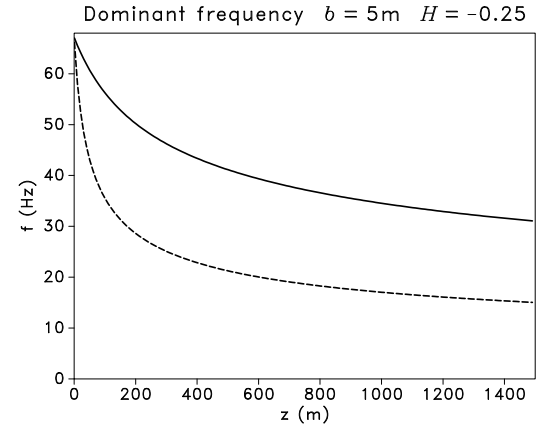

(b)

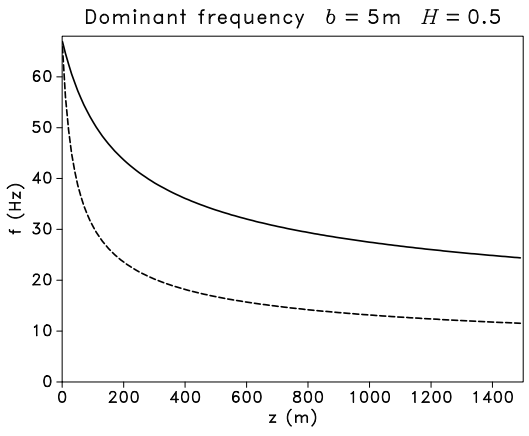

(d)

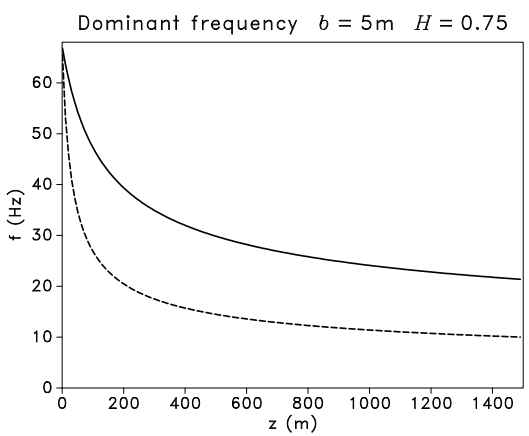

(f)

Figure 8: Evolution of the dominant frequency with depth for $\mathrm{P}$ (solid line) and $\mathrm{S}$ (dashed line) scalar waves modeled by a Ricker wavelet $\left(f_{0}=60 \mathrm{~Hz}\right)$ in heterogeneous media with $\sigma=30 \%$. For a constant exponent $H=0.25$, the dominant frequency shifts to lower frequencies faster for larger values of $b$ (a, c,e). The exponent $H$ weakly influences the evolution of the dominant frequency $(b, d, f)$. 
Several limitations should be pointed out in our study. Well logs constitute 1D samples of the geological medium in the near vertical direction. Use of 3D isotropy is the simplest assumption consistent with our limited knowledge. We consequently ignore the anisotropic effect of layering, which undoubtly affects lateral scattering when $\lambda \sim b$. Also note that the relation between fractal exponent $\beta$ and parameter $H$ depends on spatial dimension $s$. We have therefore proposed different values of parameter $H$ for depth of penetration and evolution of dominant frequency in Figures 6 and 8 and have attempted to extract some general trends.

Our analysis is limited by the fact that we used variations of the seismic velocity, but not of density, in order to be consistent with the mean field theory. This theory retrieves the Backus limit and the Rayleigh diffusion regime. Fortunately, densities commonly exhibit fewer variations. Meanwhile, analysis of the logarithm of impedance $Z$ relates directly to the reflection coefficients (Shtatland, 1991). Backscattering is known to be related to impedance fluctuations (Banik et al., 1985; Wu, 1988). Modification of the Helmholtz equation (16) in 1D to incorporate $\ln Z$ was succesfully achieved by Banik et al. (1985), and they provided a proof of O'Doherty-Anstey formula. Impedance $Z(z)$, depending on depth $z$, and reflection coefficient series $R(z)$ are connected by

$$
\begin{aligned}
\lim _{d z \rightarrow 0} R(z+d z / 2) & =\lim _{d z \rightarrow 0} \frac{Z(z+d z)-Z(z)}{Z(z+d z)+Z(z)}, \\
R(z) & =\frac{1}{2} d \ln Z(z) .
\end{aligned}
$$

Reflection series can reasonably be considered to be Gaussian and stationary only inside blocky layers, using the segmentation method (Todoeschuck et al., 1990). Incrementation of the fractional Gaussian noise, corresponding to reflection series $R(z)$, produces the non-stationary and non-Gaussian fBm describing quantity $\ln Z(z)$ (Shtatland, 1991). A white spectrum of reflectivity coefficients occurs for $\beta=2$ and generates a Brownian walk describing $\ln Z(z)$ and involving an exponential autocorrelation. We advocate the use of a non-white reflectivity hypothesis, as previously recommended by several authors (Todoeschuck et al., 1990; Lancaster and Whitcombe, 2000; Anstey and O’Doherty, 2002b).

\section{CONCLUSIONS}

The high-frequency quasi-cyclic variations of seismic velocities can be described as an antipersistent fractional Brownian motion as demonstrated by our sonic-log data from a clastic reservoir. The correlation length, estimated for von Kármán's model, is about $5 \mathrm{~m}$, but the sonic logs contain larger local cycles at 10 and $20 \mathrm{~m}$ : our method extracts one dominant cycle of deposition. Conventional seismic surveys contain frequencies as high as $100 \mathrm{~Hz}$, with typical peak frequencies at $25 \mathrm{~Hz}$. Our statistical description of geological heterogeneities below $10 \mathrm{~m}$ can thus be used consistently in our low-frequency scattering theory to estimate the scattering loss caused by smallscale heterogeneities. 
Shear waves have shorter wavelengths than compressional waves and can be more attenuated because they are more sensitive to heterogeneities. We showed the existence of a high-frequency cutoff for the depth of penetration of waves, whose position in frequency depends on the maximum size of fractal heterogeneities. The dominant frequency of a wavelet decreases faster for higher fractal exponents and for larger characteristic sizes of heterogeneities. This loss of high-frequency content influences resolution in seismic imaging. Our study recommends using low-frequency P-waves for deep targets under a strongly heterogeneous overburden.

Agreement of our results with the Backus limit and the Rayleigh diffusion regime is due to the use of velocity fluctuations. Nevertheless, proper connection with the O'Doherty-Anstey formula requires use of the logarithm of impedance. Moreover, more complex, multiple scattering occurs when sizes of heterogeneities are similar to that of the seismic wavelength. Large-scale local cycles, present in the sonic-log data, call for incorporation of resonant scattering effects into high-frequency scattering theories.

\section{ACKNOWLEDGMENTS}

We thank Apache Canada Ltd. for providing the data used in this study and Mark Tomasso from the Bureau of Economic Geology for the velocity model. We acknowledge Apache Corporation, GX Technology Corporation, and the Jackson School of Geosciences for financial support. This publication is authorized by the Director, Bureau of Economic Geology, The University of Texas at Austin.

\section{APPENDIX A}

\section{O'DOHERTY-ANSTEY FORMULA AND THE MEAN FIELD THEORY}

O'Doherty and Anstey (O'Doherty and Anstey, 1971; Resnick, 1990) proposed that local transmission coefficient $T(\omega)$ for traveltime $\Delta t$ in sedimentary layers should be

$$
T(\omega)=\exp \left[-\frac{\omega \Delta t}{2 Q(\omega)}\right]=e^{-R(\omega) \Delta t},
$$

where $R(\omega)$ is the spectrum of reflection coefficients, which is related to the spectrum of impedance fluctuations (Banik et al., 1985). Because we have used velocity fluctuations, attenuation in the dispersion relation of equation 21 depends on the velocity spectrum :

$$
\begin{aligned}
\frac{1}{Q(\omega)} & =2 \frac{\operatorname{Im}[k]}{\operatorname{Re}[k]} \\
& =k_{0}\left[E^{(1)}(0)-E^{(1)}\left(2 k_{0}\right)\right]
\end{aligned}
$$




$$
T(\omega)=\exp \left\{-\frac{k_{0} \omega}{2}\left[E^{(1)}(0)-E^{(1)}\left(2 k_{0}\right)\right] \Delta t\right\} .
$$

These formulae have been previously derived and analyzed (Lerche, 1986; Wu, 1988; Sato and Fehler, 1998). The term $E^{(1)}\left(2 k_{0}\right)$ can be interpreted as backward scattering with exchange wavenumber $2 k_{0}$, whereas the term $E^{(1)}(0)$ is forward scattering with exchange wavenumber 0 . Both energy terms reduce to $1 \mathrm{D}$ expressions because of isotropic integration, whereas one symmetry axis is imposed by propagation direction of the wave. Further interpretation can be found using a dynamic effective model for multiple scattering (Waterman and Truell, 1961): the scattered waves interfere with the main wavefield, and their relative phase continuously changes in all directions, except for significant interferences in forward and backward directions. Previous 1D derivations (Sato and Fehler, 1998) have incorporated a traveltime correction, corresponding to neglecting forward scattering in order to reproduce the O'DohertyAnstey formula. This approach extends validity of the analytical expressions to higher frequencies, but no simple traveltime phase correction exists for the mean field theory in 3D. Constant $E^{(1)}(0)$ ensures recovery of the Backus effective medium and the Rayleigh diffusion regime for very low frequencies.

\section{APPENDIX B}

\section{EXPONENTIALLY CORRELATED HETEROGENEITIES}

Results have been derived several times (Karal and Keller, 1964; Sato and Fehler, 1998) using exponential correlation function $N(r)=\exp [-r / b]$ and are added here as a specific case with simple analytical expressions within the general framework of von Kármán's media. Values of the integral in equation 22 are

$$
\begin{aligned}
S(0) & =b, \\
S\left(2 k_{0}\right) & =\frac{b}{1-2 i k_{0} b} .
\end{aligned}
$$

Using these expressions in the dispersion relation of equation 21, the new dispersion relation, phase velocity $c(\omega)$, and attenuation are

$$
\begin{aligned}
\frac{k}{k_{0}} & =\frac{c_{0}}{c(\omega)}+\frac{i}{2 Q(\omega)}=1+\frac{\sigma^{2}}{2}\left[1+\frac{\left(2 k_{0} b\right)^{2}}{1-2 i k_{0} b}\right]+O\left(\sigma^{4}\right), \\
\frac{1}{c(\omega)} & =\frac{1}{c_{0}}\left[1+\sigma^{2} \frac{1 / 2+\left(2 k_{0} b\right)^{2}}{1+\left(2 k_{0} b\right)^{2}}\right] \\
\frac{1}{Q(\omega)} & =\sigma^{2} \frac{8\left(k_{0} b\right)^{3}}{1+\left(2 k_{0} b\right)^{2}} .
\end{aligned}
$$

In the limit of very long wavelengths, i.e. $k_{0} \rightarrow 0$, attenuation and velocity reduce respectively to the Rayleigh diffusion regime and the effective medium theory of 
Backus (1962):

$$
\begin{aligned}
\frac{1}{c(\omega)} & \rightarrow \frac{1}{c_{0}}\left(1+\frac{\sigma^{2}}{2}\right), \\
\frac{1}{Q(\omega)} & \sim 8 \sigma^{2}\left(k_{0} b\right)^{3} \rightarrow 0 .
\end{aligned}
$$

\section{REFERENCES}

Anstey, N. A., and R. F. O'Doherty, 2002a, Cycles, layers, and reflections: part 1: The Leading Edge, 21, 44-51.

— 2002b, Cycles, layers, and reflections: part 2: The Leading Edge, 21, 152-158.

Backus, G. E., 1962, Long-wave elastic anisotropy produced by horizontal layering: Journal of Geophysical Research, 67, 4427-4440.

Banik, N. C., I. Lerche, and R. T. Shuey, 1985, Stratigraphic filtering, part I: derivation of the O'Doherty-Anstey formula: Geophysics, 50, 2768-2774.

Bleistein, N., J. K. Cohen, and J. W. Stockwell, 2001, Mathematics of multidimensional seismic imaging, migration, and inversion: Springer. Interdisciplinary applied mathematics.

Born, M., and E. Wolf, 1964, Principles of optics: electromagnetic, theory of propagation, interference and diffraction of light: Pergamon Press.

Chernov, L. A., 1960, Wave propagation in a random medium: McGraw-Hill.

Chesnokov, E. M., Y. A. Kukharenko, and P. Y. Kukharenko, 1998, Frequency dependence of physical parameters of microinhomogeneous media, space statistics: Revue de l'Institut Français du Pétrole, 53, 729-734.

Dolan, S. S., C. J. Bean, and B. Riollet, 1998, The broad-band fractal nature of heterogeneity in the upper crust from petrophysical logs: Geophysical Journal International, 132, 489-507.

Frankel, A., and R. W. Clayton, 1986, Finite difference simulations of seismic scattering: implications for the propagation of short period seismic waves in the crust and models of crustal heterogeneity: Journal of Geophysical Research, 91, 6465-6489.

Gist, G. A., 1994, Seismic attenuation from 3-D heterogeneities: a possible resolution of the VSP attenuation paradox: 64th Annual International Meeting, SEG, Expanded Abstracts, Society of Exploration Geophysicists, 1042-1045.

Goff, J. A., and K. Holliger, 2003, Heterogeneity in the crust and upper mantle: nature, scaling, and seismic properties: Kluwer Academic/Plenum Publishers.

Goff, J. A., and T. H. Jordan, 1988, Stochastic modeling of seafloor morphology: inversion of sea beam data for second-order statistics: Journal of Geophysical Research, 93, 13,589-13,608.

Golub, G. H., and V. Pereira, 1973, The differentiation of pseudo-inverse and nonlinear least squares problems whose variables separate: SIAM Journal of Numerical Analysis, 10, 413-432.

Haney, M. M., K. van Wijk, and R. Snieder, 2005, Radiative transfer in layered media and its connection to the O'Doherty-Anstey formula: Geophysics, 70, no. 1, $\mathrm{T} 1-\mathrm{T} 11$. 
Hewett, T. A., 1986, Fractal distributions of reservoir heterogeneity and their influence on fluid transport: Presented at the 61st Technical Conference, Society of Petroleum Engineering, Society of Petroleum Engineering, SPE 15386.

Holliger, K., and A. R. Levander, 1992, A stochastic view of lower crustal fabric based on evidence from the Ivrea zone: Geophysical Research Letters, 19, 1153-1156.

Hurst, H. E., 1951, Long-term storage capacity of reservoirs: Transaction of American Society of Civil Engineering, 116, 770-808.

Kanaun, S. K., and V. Levin, 2008, Self-consistent methods for composites, vol.2: wave propagation in heterogeneous materials: Springer-Verlag.

Karal, F. C., and J. B. Keller, 1964, Elastic, electromagnetic, and other waves in a random medium: Journal of Mathematical Physics, 5, 537-547.

Klimeš, L., 2002, Correlation functions of random media: Pure and Applied Geophysics, 159, $1811-1831$.

Lancaster, S., and D. Whitcombe, 2000, Fast-track 'coloured' inversion: 60th Annual International Meeting, SEG, Expanded Abstracts, Society of Exploration Geophysicists, $1572-1575$.

Le Gonidec, Y., and D. Gibert, 2007, Multiscale analysis of waves reflected by granular media: acoustic experiments on glass beads and effective medium theories: Journal of Geophysical Research, 112.

Lerche, I., 1986, Stratigraphic filter theory: combined effects of parallel bedding and random inhomogeneities: Geophysics, 51, 1531-1537.

Li, C. F., 2003, Rescaled-range and power spectrum analyses on well-logging data: Geophysical Journal International, 153, 201-212.

Lord, R. D., 1954, The use of the Hankel transform in statistics I. General theory and examples: Biometrika, 41, 44-55.

Lu, S., F. J. Molz, G. E. Fogg, and J. W. Castle, 2002, Combining stochastic facies and fractal models for representing natural heterogeneity: Hydrogeology Journal, 10, $475-482$.

Mandelbrot, B. B., 1983, The fractal geometry of nature: W. H. Freeman and Co.

Mandelbrot, B. B., and J. R. Wallis, 1969, Some long-run properties of geophysical records: Water Resources Research, 5, 321-340.

Marsan, D., and C. J. Bean, 2003, Multifractal modeling and analyses of crustal heterogeneity, in Heterogeneity in the crust and upper mantle: nature, scaling, and seismic properties: Kluwer Academic/Plenum Publishers, 207-236.

Morlet, J., G. Arens, E. Fourgeau, and D. Giard, 1982, Wave propagation and sampling theory - part I: complex signal and scattering in multilayered media: Geophysics, 47, 203-221.

Müller, T. M., and S. A. Shapiro, 2001, Most probable seismic pulses in single realizations of two- and three-dimensional random media: Geophysical Journal International, 144, 83-95.

O'Doherty, R. F., and N. A. Anstey, 1971, Reflections on amplitudes: Geophysical Prospecting, 19, 430-458.

Painter, S., and L. Paterson, 1994, Fractional Lévy motion as a model for spatial variability in sedimentary rocks: Geophysical Research Letters, 21, 2857-2860.

Resnick, J. R., 1990, Stratigraphic filtering: Pure and Applied Geophysics, 132, 49- 
65.

Rivera, N. A., S. Ray, J. L. Jensen, A. K. Chan, and W. B. Ayers, 2004, Detection of cyclic patterns using the wavelets: an example study in the Ormskirk sandstone, Irish sea: Mathematical Geology, 36, 529-543.

Rytov, S. M., Y. A. Kravtsov, and V. I. Tatarski, 1989, Wave propagation through random media: Springer-Verlag. Principles of statistical radio physics.

Sato, H., and M. Fehler, 1998, Seismic wave propagation and scattering in the heterogeneous Earth: Springer-Verlag.

Schottky, W., 1926, Small-shot effect and Flicker effect: Physical Reviews, 28, 74104.

Shapiro, S. A., and P. Hubral, 1999, Elastic waves in random media: Springer-Verlag.

Shapiro, S. A., and H. Zien, 1993, The O'Doherty-Anstey formula and localization of seismic waves: Geophysics, 58, 736-740.

Sheng, P., B. White, Z. Q. Zhang, and G. Papanicolaou, 1986, Multiple scattering noise in one dimension: universality through localization length scaling: Physical Review Letters, 57, 1000-1003.

Shiomi, K., H. Sato, and M. Ohtake, 1997, Broad-band power-law spectra of well log data in Japan: Geophysical Journal International, 130, 57-64.

Shtatland, E. S., 1991, Fractal stochastic models for acoustic impedance: an explanation of scaling or 1/f geology and stochastic inversion: 51st Annual International Meeting, SEG, Expanded Abstracts, Society of Exploration Geophysicists, 15981601.

Stefani, J., and S. D. Gopa, 2001, On the power-law behavior of subsurface heterogeneity: 61st Annual International Meeting, SEG, Expanded Abstracts, Society of Exploration Geophysicists, 2033-2036.

Stovas, A., and B. Ursin, 2007, Equivalent time-average and effective medium for periodic layers: Geophysical Prospecting, 55, 871-882.

Tatarski, V. I., 1961, Wave propagation in a turbulent medium: McGraw-Hill.

Todoeschuck, J. P., O. G. Jensen, and S. Labonte, 1990, Gaussian scaling noise model of seismic reflection sequences: evidence from well logs: Geophysics, 55, 480-484.

Turcotte, D. L., 1997, Fractals and chaos in geology and geophysics: Cambridge University Press.

Uscinski, N. J., 1977, The elements of wave propagation in random media: McGrawHill.

van der Baan, M., 2001, Acoustic wave propagation in one-dimensional random media: the wave localization approach: Geophysical Journal International, 145, 631-646.

van der Baan, M., J. Wookey, and D. Smit, 2007, Stratigraphic filtering and source penetration depth: Geophysical Prospecting, 55, 679-684.

von Kármán, T., 1948, Progress in the statistical theory of turbulence: Journal of Marine Research, 7, 252-264.

Walden, A. T., and J. W. J. Hosken, 1985, An investigation of the spectral properties of primary reflection coefficients: Geophysical Prospecting, 33, 400-435.

Waterman, P. C., and R. Truell, 1961, Multiple scattering of waves: Journal of Mathematical Physics, 2, 512-537.

White, B., P. Sheng, and B. Nair, 1990, Localization and backscattering spectrum of 
seismic waves in stratified lithology: Geophysics, 55, 1158-1165.

Wu, R. S., 1982, Attenuation of short period seismic waves due to scattering: Geophysical Research Letters, 9, 9-12.

— , 1988, Discussion on: "Stratigraphic filter theory: combined effects of parallel bedding and random inhomogeneities," I. Lerche, author: Geophysics, 53, 995-997.

_ 1993, Separation of scattering and absorption in 1-D random media from spatio-temporal distribution of seismic energy: 53rd Annual International Meeting, SEG, Expanded Abstracts, Society of Exploration Geophysicists, 1014-1017.

Wu, R. S., and K. Aki, 1985, Elastic wave scattering by a random medium and the small scale inhomogeneities in the lithosphere: Journal of Geophysical Research, 90, 10261-10273.

Ziolkowski, A., P. Hanssen, R. Gatliff, H. Jakubowicz, A. Dobson, G. Hampson, X. Y. $\mathrm{Li}$, and E. Liu, 2003, Use of low frequencies for sub-basalt imaging: Geophysical Prospecting, 51, 169-182. 